\title{
L'errance diagnostique : le point de vue des malades
}

Christian Cottet

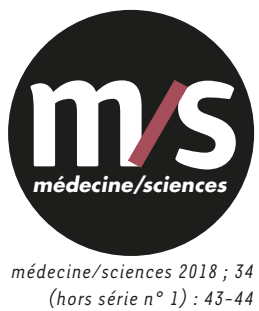

> C'est également en tant que père d'une jeune femme atteinte d'amyotrophie spinale que je m'exprime aujourd'hui.

L'errance diagnostique est une véritable souffrance pour les malades et les familles. Celle-ci fait référence à la période entre les premiers symptômes d'une maladie et son diagnostic confirmé. L'alliance Maladies rares a réalisé une enquête, qui a démontré que plus d'un quart des malades atteints de maladies rares attendent près de quatre ans pour que la recherche de leur diagnostic débute. Une fois dans le circuit du parcours diagnostic, cette recherche dure au moins un an et demi pour une grande majorité de malades et dépasse 5 ans pour plus d'un quart d'entre eux. La première période sensible est celle qui s'écoule entre les premiers symptômes et une orientation vers un centre spécialisé. Auparavant, un quart des malades attendait plus de quatre ans, contre deux ans au cours des dernières années. II s'agit d'une amélioration, bien que ce délai demeure encore trop long. La seconde période est celle qui s'étend du premier contact avec des professionnels appropriés et le diagnostic final. Elle renvoie à l'organisation du système de santé issue, notamment, des premiers plans Maladies rares, et de sa performance en matière de parcours diagnostic. On considère aujourd'hui que, in fine, la moitié des malades atteints d'une maladie rare ne dispose pas d'un diagnostic précis.

Cette errance est responsable d'un gaspillage de ressources médicales. Un quart des malades ont consulté au moins cinq médecins avant d'obtenir leur diagnostic, dans l'étude menée par l'Alliance Maladies Rares. Elle est également source d'angoisse pour les familles, et d'aggravation de l'état des malades. Elle entretient un cercle vicieux, en retardant les possibilités de conseil génétique tant pour le malade adulte, les parents de l'enfant malade que pour les autres membres de leur famille.

Après deux plans de santé publics pour les maladies rares et le lancement du plan France Médecine Génomique, les connaissances, outils et infrastructures sont en place ou en cours de mise en place. Avec ces outils, que faut-il faire? C'est ce que nous proposons pour le PNMR3: une mobilisation générale contre l'errance diagnostique, avec un plan d'action structuré en trois parties.

L'action I vise à poursuivre la cartographie et organiser la complémentarité des expertises diagnostiques. II s'agit avant tout de mobiliser les filières de santé et les laboratoires de diagnostics, afin de cartographier et organiser nationalement l'articulation et la complémentarité des champs d'expertise pour les diagnostics, de manière exhaustive. $\varepsilon n$ effet, les champs d'expertise diagnostics sont nombreux : biochimie, biologie, anatomo-pathologie, électrophysiologie, imagerie, génétique, génomique...

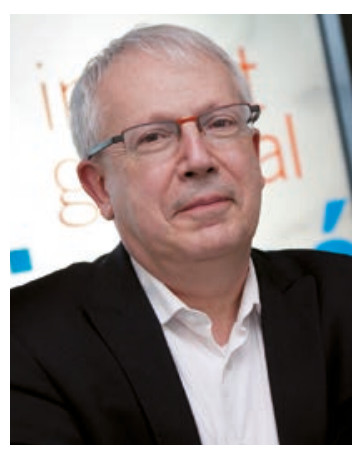

Directeur général de l'AFMTéléthon, AFM-Téléthon, 1 , rue de l'Internationale, 91000 Évry, France. ccottet@afm-telethon.fr

En parallèle du développement du whole exome sequencing et du whole genome sequencing, l'utilisation des panels de gènes se poursuit. Il existe un besoin impératif d'organisation et de visibilité des acteurs au niveau des laboratoires de diagnostic, et d'autant plus en raison de l'implémentation prochaine des plateformes nationales de séquençage. Au-delà de la génétique, ce travail d'inventaire, de cartographie et d'harmonisation est également nécessaire pour les autres champs d'expertise diagnostique. Des filières ont commencé cet état des lieux. Il faut le poursuivre, et harmoniser en inter-filières pour rendre lisible et efficace le parcours diagnostic. II faut aussi poursuivre la formalisation et la publication des arbres de décision complets des parcours diagnostics.

L'objectif est donc d'harmoniser et de rendre lisible au plan national l'organisation des plateformes diagnostiques, la répartition des compétences, et formaliser les parcours diagnostics, c'est-à-dire l'articulation logique des champs de compétence.

Ce plan devra garantir une prise en charge financière de ces examens et une équité territoriale.

Dans le cadre de l'action $n^{\circ} 2$, le plan Médecine Génomique 2025 doit être déployé rapidement et adapté à l'organisation du système de santé pour les maladies rares. Ce plan vise essentiellement à offrir à la France une capacité de séquençage très haut débit de type industriel. Les moyens apportés ont pour objectif de faire fonctionner ces plateformes au niveau analytique et post-analytique.

La gouvernance du plan France Médecine Génomique devra intégrer un représentant du comité de diagnostic des maladies rares, afin de s'assurer d'une parfaite cohérence entre les deux plans de santé publique. 
Si ces moyens permettent de doter la France d'une capacité de réalisation de génomes complets, toutes les demandes ne pourront néanmoins être réalisées. L'indication d'une telle analyse devra être justifiée. II est en effet prévu dans le plan, la possibilité d'analyser sur ces plateformes 20000 génomes de patients atteints de maladies rares et de leurs familles, soit environ 60000 génomes dans le cadre de stratégies trio.

Ce projet devra s'articuler avec les organisations existantes. Les filières de santé maladies rares et le réseau NGS diagnostic pourront faciliter ces interactions.

II devrait être proposé des réunions de concertation pluridisciplinaires RCP d'entrée et de sortie.

Il est donc nécessaire de prioriser les indications de séquençage de type WES et WGS, organiser la collaboration et les complémentarités entre les médecins des centres de référence et de compétence, les généticiens et cliniciens hospitaliers, et les plateformes de séquençage, organiser le parcours de l'information et le retour du diagnostic au malade. II s'agit d'organiser les compétences de chacun et leur complémentarité, pour que le parcours du pré-analytique, de l'analytique et du post-analytique pour l'interprétation soit fluide et lisible.

Enfin, l'action $n^{\circ} 3$ vise à lancer un grand plan d'action mobilisateur, « un diagnostic pour chacun », impliquant la création d'un comité du diagnostic. Il s'agit de mobiliser tous les acteurs, dans le cadre d'une action concernée: les filières de santé, les centres de référence et de compétences, les plateformes de diagnostics, les associations de malades, les réseaux d'accompagnement des malades, reprendre chaque dossier et faire un point du parcours de diagnostic de chaque malade pour ne pas laisser au bord de la route des patients non avertis des progrès en matière de diagnostics. II s'agit de vérifier, avec le malade, si les étapes incontournables du chemin du diagnostic ont bien été suivies. Dans la négative, il est proposé de reprendre le parcours du diagnostic pour mener à bien toutes les étapes indispensables.

Le déploiement de cette action peut se faire dans le cadre des FSMR qui coordonneront l'action des centres de référence et de compétences, mais aussi mobiliseront les généralistes et spécialistes de la médecine de ville, car il faut aussi décliner cette approche (peut-être dans un deuxième temps) à la population de malades atteints de maladies rares qui ne sont pas aujourd'hui suivies par un centre de référence/compétence ou dans une filière de santé spécifique.

Enfin, nous proposons la création d'un comité du diagnostic au niveau du PNMR3, pour assurer une veille technologie, clinique et éthique autour de la question centrale du diagnostic. Ce comité donnera l'impulsion. Il vérifiera qu'il n'existe pas de perte de chances au niveau du diagnostic et adaptera en permanence les moyens et les outils. II devra mettre en place et gérer les tableaux de bord d'évolution de la situation de diagnostic en France. II assurera le lien avec la recherche génomique et thérapeutique en identifiant les patients éligibles aux biothérapies innovantes en interface avec les acteurs concernés.

En conclusion, quel est l'objectif à atteindre?

- Zéro patient en situation d'errance diagnostique à 5 ans.

- Passer dans les cinq ans de la notion d'errance diagnostique à celle d'impasse diagnostique.

À l'issue du PNMR 3, les patients ne disposant pas d'un diagnostic précis pourraient se limiter à ceux pour lesquels le développement de la science et des techniques ne permet pas d'aboutir à un diagnostic précis.

Du côté génétique, tous les patients devront avoir accès à une analyse de type WES ou WGS, si les études préalables sont restées négatives. $\diamond$

Diagnostic delay in the field of rare diseases

from a patient organisation perspective

\section{LIENS D'INTÉRÊT}

L'auteur déclare n'avoir aucun lien d'intérêt concernant les données publiées dans cet article. 